Cumhuriyet International Journal of Education-CIJE

e-ISSN: 2147-1606

Vol 4 (3), 2015, $47-61$

\title{
Determination of the Opinions of the Prospective Teachers With Regards To the Science Special Field Qualifications
}

\author{
Tolga BABACAN, Fatma ŞAŞMAZ ÖREN
}

Summary

\section{Introduction}

The fact that Turkey is below the average level of other OECD countries according to the PISA (The Program for International Student Assessment) 2009 assessment report, as organized by the OECD (Organization for Economic Cooperation and Development), to which Turkey is a member too, led to questioning of the skills necessary to be possessed by teachers. The "General Skills of Teaching Profession" created by a committee formed from Ministry of National Education (MNE) and Higher Education Institutions was published in 2008 in relation to the characteristics necessary to be present in teachers (MNE, 2008). All documentation prepared previously was examined during this study and teacher profession skills of Great Britain, USA, Seychelles, Australia, and Ireland were studied in order to obtain a general understanding, as a result of which 6 main skills, 31 sub-skills and 233 performance skills were identified (MNE, 2008). Accordingly, the skill fields are "personal and professional values - professional growth", "knowing the student", "teaching and learning process", "monitoring and assessment of learning, development", "school, family and social relations", and "knowledge of program and contents". Natural Sciences and Technology Education Special Field Skills were identified during the same study. The developed Natural Sciences and Technology / Natural Sciences Education special field skills consist of 5 skill areas and scopes explaining the contents of these fields, 24 sub-skills, as well as 132 performance indicators denoted as A1, A2, A3. Identifying the characteristics needed in a qualified teacher being trained at the Department of Natural Sciences Education gains importance in terms of Turkey's ascension to higher levels in international evaluation studies that Turkey participates in. In this regard, this study intends to gather the views of prospective teachers receiving training at the Department of Natural Sciences Education regarding the proficiency in their fields. Therefore, the subject of the study can be expressed as follows: "What are the views of prospective teachers in relation to special field proficiency in Natural Sciences and technology/Natural Sciences Education?".

\section{Method}

This study, conducted in order to identify the views of prospective teachers enrolled in the Natural Sciences and Technology / Natural Sciences Education program about the Natural Sciences and Technology Special Field Proficiency, is defined as a qualitative research. The study was conducted on 4th grade students of the Natural Sciences Education Program of Celal Bayar University Faculty of Education during the fall semester of 2015-2015 academic years. The interview form, which was used as data collection tool during the study, was applied to 105 prospective teachers. Semi-structured interviews, which are another data collection tool in this study, were conducted with 13 prospective teachers. Data obtained from the study was analyzed by means of content analysis. Data obtained from interview for was presented in the form of a table and supported by direct citing from the semi-structured interviews.

\section{Findings}

According to the findings obtained during the study, prospective teachers consider themselves to possess the average level of proficiency in Special Fields of Natural Sciences and Technology / Natural Sciences Education. It was found that a significant ratio of prospective 
teachers participating in the study understand the Special Field Proficiency in Natural Sciences and Technology Education to be the field knowledge (physics, chemistry, biology). Moreover, it was found that of the six special field dimensions prospective teachers gave their views within the dimension of "planning and organizing the teaching-learning process" at most. Therewith, a conclusion was made that although prospective teachers provided views on the five of six special field proficiencies; no views were reported on the proficiency of "cooperation in school, family and social environment".

\section{Discussion and Conclusion}

When the views of prospective teachers participating in the study were examined, it was determined that prospective teachers viewed the subject field knowledge as the primary special field proficiency. One of the reasons is the fact that classes taught within the scope of Natural Sciences Education programs of universities are more related to field knowledge (Physics, Chemistry, Biology), whereas classes related to special fields are very few, only the Special Teaching Methods classes cover the special field proficiencies. One of the important conclusions of this study is the fact that prospective teachers expressed no views on the "cooperation in family, school and social environment" special field proficiency. One of the reasons for such conclusions in studies performed is that prospective teachers consider other special field proficiencies to be of more importance while such proficiencies as school culture, social leadership and cooperation in families are regarded as matters of secondary importance. In conclusion, when the study is reviewed, it can be said that prospective teachers consider themselves to have the average level of special field proficiency, whilst not entirely knowing what the special field proficiencies include in actuality. In this regard, it can be said that especially the natural sciences and technology education programs of universities need to be revised. More qualified teachers in the field can be raised by creating a new natural sciences and technology education program that would include particularly the proficiency of cooperation in school, family and social environment among other special field proficiencies. 